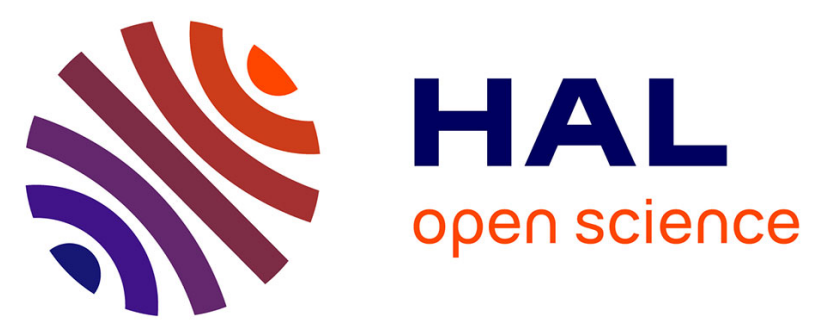

\title{
The effect of strain biaxiality on the fracture of zirconium alloy fuel cladding
}

Ahmed Zouari, Matthew Bono, David Leboulch, Thomas Le Jolu, Jérôme Crepin, Jacques Besson

\section{To cite this version:}

Ahmed Zouari, Matthew Bono, David Leboulch, Thomas Le Jolu, Jérôme Crepin, et al.. The effect of strain biaxiality on the fracture of zirconium alloy fuel cladding. Journal of Nuclear Materials, 2021, pp.153070. 10.1016/j.jnucmat.2021.153070 . cea-03330340

\section{HAL Id: cea-03330340 https: / hal-cea.archives-ouvertes.fr/cea-03330340}

Submitted on 31 Aug 2021

HAL is a multi-disciplinary open access archive for the deposit and dissemination of scientific research documents, whether they are published or not. The documents may come from teaching and research institutions in France or abroad, or from public or private research centers.
L'archive ouverte pluridisciplinaire HAL, est destinée au dépôt et à la diffusion de documents scientifiques de niveau recherche, publiés ou non, émanant des établissements d'enseignement et de recherche français ou étrangers, des laboratoires publics ou privés.

\section{(1) (1) $\$$}

Distributed under a Creative Commons Attribution - NonCommercial - NoDerivatives| 4.0 


\title{
The effect of strain biaxiality on the fracture of zirconium alloy fuel cladding
}

\author{
Journal of Nuclear Materials 554 (2021) 153070
}

The published journal article is available online: https://doi.org/10.1016/j.jnucmat.2021.153070

\author{
Ahmed Zouari \\ Email : Ahmed.zouari@mines-paristech.fr \\ Université Paris-Saclay, CEA, Service d'Etude des Matériaux Irradiés, 91191, Gif-sur-Yvette, France \\ Mines ParisTech - PSL Research University, UMR CNRS 7633, Centre des Matériaux, 91003 Evry, France \\ Matthew Bono \\ Email : Matthew.bono@cea.fr \\ Université Paris-Saclay, CEA, Service d'Etude des Matériaux Irradiés, 91191, Gif-sur-Yvette, France \\ David Le Boulch \\ Email : David.leboulch@cea.fr \\ Université Paris-Saclay, CEA, Service d'Etude des Matériaux Irradiés, 91191, Gif-sur-Yvette, France \\ Thomas Le Jolu \\ Email : Thomas.lejolu@cea.fr \\ Université Paris-Saclay, CEA, Service d'Etude des Matériaux Irradiés, 91191, Gif-sur-Yvette, France \\ Jérôme Crépin \\ Email : Jerome.crepin@mines-paristech.fr \\ Mines ParisTech - PSL Research University, UMR CNRS 7633, Centre des Matériaux, 91003 Evry, France \\ Jaques Besson \\ Email : Jacques.besson@mines-paristech.fr \\ Mines ParisTech - PSL Research University, UMR CNRS 7633, Centre des Matériaux, 91003 Evry, France
}

\section{Corresponding author}

Ahmed Zouari

Email : Ahmed.zouari@mines-paristech.fr

\begin{abstract}
During a Reactivity Initiated Accident (RIA), nuclear fuel cladding experiences a multiaxial loading state in which the Pellet-Cladding Mechanical Interaction (PCMI) produces a strain biaxiality ratio $\left(\varepsilon_{\mathrm{zz}} / \varepsilon_{\theta \theta}\right)$ of between 0 and 1 . This study examines the effect of strain state and loading path on the fracture of Zircaloy-4 fuel cladding. A new mechanical test has been developed in order to apply several levels of strain biaxiality. Digital Image Correlation (DIC) is used to measure the strain field, to identify the onset of cladding failure, and to obtain the corresponding critical loading state. Results show that the strain biaxiality has a significant effect on the hoop strain at failure, and the smallest fracture strain is obtained for nearly plane strain conditions. A model is proposed for the accumulation of damage due to plastic deformation, based on the Lode parameter of the plastic strain rate tensor. The model is used to predict the failure hoop strain as a function of the strain biaxiality ratio $\left(\varepsilon_{z z} / \varepsilon_{\theta \theta}\right)$, and good agreement is found between the experimentally measured and predicted failure strains.
\end{abstract}




\section{Introduction}

A Reactivity Initiated Accident (RIA) caused by the ejection of a control rod is a design basis accident that can potentially occur in a Pressurized Water Reactor (PWR). During the first phase of a RIA, the zirconium alloy fuel cladding tubes may be subjected to Pellet-Cladding Mechanical Interaction (PCMI) due to the thermal expansion of the pellet. In this scenario, the expanding pellet imposes a hoop strain and a tensile axial strain on the fuel cladding. Data from previous experiments in research reactor programs simulating the PCMI phase of RIAs show that, at high burnup of the fuel, the cladding undergoes a multiaxial loading state characterized by a strain biaxiality ratio $\left(\varepsilon_{\mathrm{ZZ}} / \varepsilon_{\theta \theta}\right)$ between plane-strain (no axial strain in the cladding) and equi-biaxial tension (equal tensile strains in the hoop and axial directions). These values were obtained from measurements of the residual axial and hoop strains on cladding tubes [1].

Additional experiments are necessary to characterize the failure conditions of fuel cladding in accidental scenarios. However, due to the high cost and complexity of tests in experimental reactors, it would be preferable to obtain the necessary information from laboratory tests that allow the effects of various parameters to be studied individually. To understand the effect of the strain biaxiality ratio $\left(\varepsilon_{z z} / \varepsilon_{\theta \theta}\right)$ on the hoop strain at fracture of the cladding, laboratory tests should accurately reproduce reactor conditions that affect this parameter. The objective of this study is to develop a laboratory test that reproduces the strain biaxiality imposed on the cladding in the reactor, and to analyze its effect on the hoop strain at failure of non-irradiated Cold-Worked Stress-Relieved (CWSR) Zircaloy-4 cladding. Tests using different strain ratios were coupled with a ductile failure model to examine how the failure strain and failure mechanism are affected by the strain biaxiality ratio.

Previous researchers have used various testing methods to evaluate the effect of strain biaxiality on the fracture behavior of zirconium alloys. The effect of the strain biaxiality ratio for sheet metals has been studied by Yunchang and Koss [2], Seo et al. [3] and Kim et al. [4]. Yunchang and Koss [2] performed biaxial tests that showed a decrease in the ductility of hydrided Zircaloy-2 sheets as the strain biaxiality increases. The ductility decrease is more pronounced for high hydride concentrations. However, Seo et al. [3] and Kim et al. [4] found either no variation or a slight increase of the ductility when the biaxiality increases from 0 to 1 for Zircaloy-4 and Zirlo sheets, respectively. Each of these studies [2-4] found that the failure strain of sheets is much higher in pure tension (strain biaxiality $\approx-0.5$ ) than in plain strain (strain biaxiality $\approx 0$ ). These studies have revealed that the strain biaxiality ratio is an important parameter with a significant effect on the strain at failure. However, these results were obtained for sheet materials and cannot be directly applied to cladding tubes, which have different crystallographic texture and mechanical properties.

The effect of biaxiality on the failure of cladding specimens has been studied by many authors with different loading modes. Kaplar et al [5] examined the impact of biaxiality in plane strain and equi-biaxial conditions for $\mathrm{Zr}-1 \% \mathrm{Nb}$ alloy. In their tests, fuel cladding tubes were loaded with a combination of internal pressure and axial tensile force to obtain different strain biaxialities. Their study shows that an increase of the strain ratio $\left(\varepsilon_{\mathrm{zz}} / \varepsilon_{\theta \theta}\right)$ from 0 to 1 leads to a $50 \%$ reduction of the hoop strain at failure. In these tests, the tube fails by the onset of plastic instabilities in the form of ballooning followed by localized necking [6]. This type of loading does not reproduce the displacement-controlled strain imposed on the cladding by the expanding fuel pellet, and thus the test does not reproduce PCMI conditions.

Very few mechanical tests can load tubular samples with a high-biaxiality displacementcontrolled load. One of the tests that best represents PCMI involves placing a pellet-shaped cylindrical device inside a sample of fuel cladding. The device is compressed axially, causing 
it to expand in diameter, thereby creating a hoop strain in the cladding sample (Fig. 1a). Mishima [7] developed a test in which a ductile, pellet-shaped lead cylinder was compressed by two pistons to measure the failure hoop strain of beryllium cladding tubes. Researchers have also used a segmented expanding mandrel with an axial core of ductile material [8]. Compression of the ductile core causes the segmented mandrel to expand, thereby imposing a hoop strain on the sample. Expansion Due to Compression (EDC) tests have been the subject of various recent numerical and experimental studies [9-14] due to their ability to characterize the loading and the failure mode encountered during the PCMI stage of RIAs.

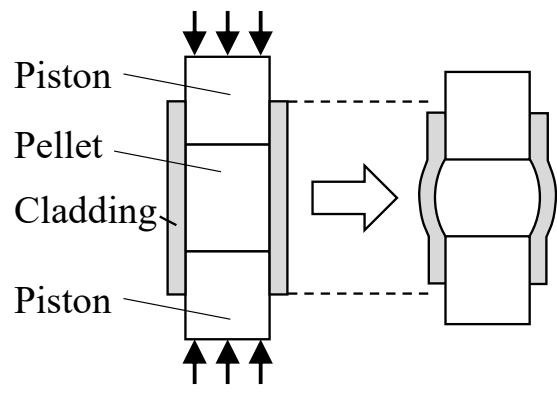

(a)

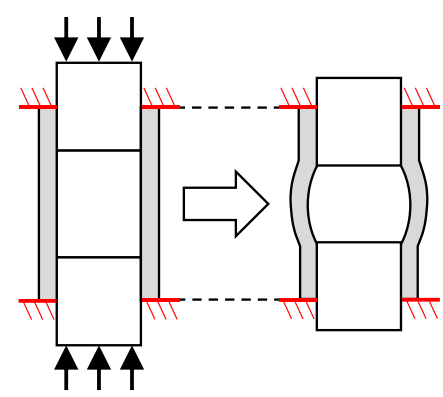

(b)

Fig. 1. EDC test configuration, (a) free-end EDC test, (b) fixed-end EDC test

In the original version of the EDC test, the ends of the tubular sample are free and unconstrained. During this type of free-end EDC test, the sample shrinks axially as it expands in diameter (Fig. 1a). Therefore, the strain biaxiality in the sample, $\left(\varepsilon_{z z} / \varepsilon_{\theta \theta}\right)$, is not representative of that experienced by fuel cladding during PCMI. In order to produce a more representative biaxial strain state in the sample, Nobrega [15] introduced two axial retainers at the ends of the tube to restrain it from axial shrinkage (Fig. 1b). The end restraining fixture prevents any change in length of the sample, so the strain biaxiality is much more representative than that of a freeend test. Hellouin de Menibus et al [16] used this type of fixed-end EDC test on Zircaloy-4 cladding at $25^{\circ} \mathrm{C}$ and found that the fracture strain is half of that obtained in free-end EDC tests.

Shinozaki et al. [17-18] and Li et al. [19] developed a type of EDC test in which the sample experiences tensile axial strain as well as tensile hoop strain. Therefore, the strain biaxiality is very similar to that experienced by fuel cladding during PCMI. This test uses two actuators to load the sample. The first applies an axial tensile load to the ends of the tube, and the second compresses a media inside the tube to impose a hoop strain. They performed tests on pre-cracked cladding samples with an outer hydride rim, and they measured the strains using strain gauges mounted on the side of the tube opposite the pre-crack. Their research revealed that the rupture strain of a pre-cracked cladding tube decreases as strain biaxiality increases. In their case, the rupture is governed by the propagation of an existing crack, which they analyzed by calculating the $J$ integral.

In order to be able to test the rupture strain of irradiated cladding tubes in conditions representative of the PCMI phase of an RIA, a research program is ongoing at the French Institut Tripartite CEA-EDF-Framatome. An experimental device that can apply a displacementcontrolled biaxial load is being developed at the CEA for application in a hot cell. This Expansion Due to Compression with Tension (EDCT) device is used in the current study to determine the effect of the biaxiality on the fracture strain, and the results are coupled with a numerical procedure to analyze the ductile damage. The EDCT device was used in this study to perform tests on CWSR fuel cladding samples. The initial tests were performed on nonirradiated samples at room temperature with a slow strain rate. The current paper describes the 
results and analysis of these tests. Based on the results, future efforts will be directed at adapting the device to allow testing at higher temperatures, greater strain rates, and implementation in a hot cell.

The new EDCT device and the strain measurement technique are described in Section 2. In Section 3, the results of the mechanical tests are presented. The influence of biaxiality on the fracture strain is studied using free-end and fixed-end EDC tests in addition to EDCT tests. Scanning electron microscopy (SEM) observations of the fracture surfaces are also presented. In Section 4, the tests are simulated using the finite element method (FEM). Section 5 presents a fracture initiation model that reproduces the experimental results by post-processing elastoplastic finite element simulations.

\section{Experimental procedures}

The material used in this study was non-irradiated CWSR Zircaloy-4 fuel cladding, which is commonly used in PWRs. The tubes have an outer diameter of $9.5 \mathrm{~mm}$, an inner diameter of $8.36 \mathrm{~mm}$, a wall thickness of $0.57 \mathrm{~mm}$, and a length of $27 \mathrm{~mm}$. In order to ensure that fracture occurred in the central part of the sample, some samples were prepared with a wall thickness that was reduced to $0.52 \mathrm{~mm}$ in this region. The pellet inside the sample is made of polytetrafluoroethylene (PTFE) and has a length of $14 \mathrm{~mm}$ and a diameter of $8.34 \mathrm{~mm}$.

Three types of EDC tests were used to obtain different strain biaxialities, which consequently affects the stress state. The tests were designed to obtain a mean hoop strain rate of approximately $10^{-4} \mathrm{~s}^{-1}$ in the area of interest. All tests were conducted up to failure, except the free-end EDC test, which did not rupture. The tests were carried out at room temperature on an electro-mechanical tensile testing machine equipped with a $100 \mathrm{kN}$ load cell and displacement sensors.

\subsection{Free-end and fixed-end EDC tests}

In this study, EDC tests were performed using a compression cage on a tensile testing machine. The free-end EDC test consists of axially compressing, between two pistons, a ductile PTFE pellet inside a sample of cladding tube with free ends, as shown in Fig. 1a. The axial compression causes the pellet to expand in diameter, thereby imposing a hoop strain on the cladding in the area in contact with the pellet, the area of interest. The test reproduces the displacement-controlled loading mode of PCMI, in which the expanding fuel pellet imposes a strain on the fuel cladding. However, at the beginning of the test, the sample shrinks axially as it expands in diameter, such that $\varepsilon_{z z} / \varepsilon_{\theta \theta} \approx-0.6$ and $\sigma_{z z} / \sigma_{\theta \theta} \approx 0$. Thus, the biaxiality created in this test is not representative of the PCMI phase.

The fixed-end EDC test (Fig. 1b) is an improved version of the free-end EDC test. The tubular sample passes through the plates of an end restraining fixture, and the ends are fixed in place. The samples used in the current study are similar to those used by Hellouin de Menibus et al. [16]. Each sample consists of a tube with a threaded cap welded to each end. Prior to welding the caps in place, a ductile pellet and two stainless steel disks are inserted inside the sample. After passing the ends of the sample through the holes in the restraining fixture, a nut is screwed onto each threaded cap. Each threaded cap contains a central hole so that a piston can pass through it in order to compress the pellet. At the beginning of the test, the strain state resembles that of a free-end EDC test. However, as the test progresses, the strain biaxiality approaches plane-strain $\left(\varepsilon_{z z} / \varepsilon_{\theta \theta} \approx 0\right)$. Thus, the biaxiality is more representative than that of a free-end test, and it approaches the lower limit of the PCMI phase near the end of the test. 


\subsection{EDC test with Tensile load (EDCT)}

In order to reach higher biaxiality levels, a type of EDC test with an axial tensile load on a zirconium alloy sample was developed in this study. Fig. 2 presents a schematic illustration of the test.

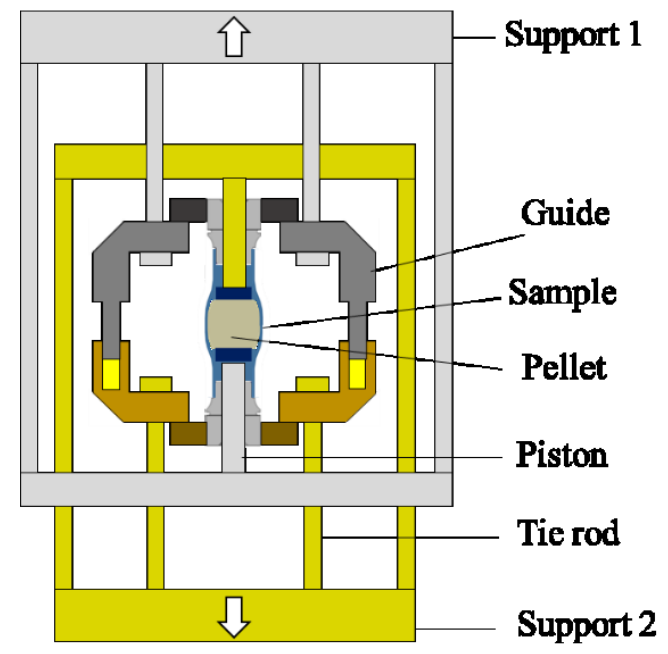

Fig. 2. EDC test with a tensile load

During this test, in addition to the compression of the pellet, four deformable tie rods (two on each side), which are attached to the outer plates of the compression cage, apply a tensile load to the ends of the sample. Therefore, the sample is subjected to simultaneous tensile hoop and axial loads during the test. The strain biaxiality, $\varepsilon_{\mathrm{zz}} / \varepsilon_{\theta \theta}$, in this test is controlled by the mechanical behavior of the tie rods. These tie rods are made of stainless steel and are designed to deform plastically during the test. The force that they apply to the sample varies throughout the test and is a function of their geometry and the mechanical tensile behavior of the stainless steel, namely the yield strength and the strain hardening characteristics. Finite element modeling was used to identify a suitable material and geometry for the tie rods in order to obtain a strain biaxiality close to 0 . Thereafter, the cross section of the ties rods was altered slightly to either increase or decrease the biaxiality.

\subsection{Strain measurement technique}

Strain measurements on the outer surface of the tube were performed with a digital image correlation (DIC) system (Fig. 3). The surfaces of the specimens were spray-painted with a random black speckle pattern on a uniform white background, and the samples were illuminated with two spotlights. The DIC system is equipped with 2 cameras with 4 megapixel resolution, a focal length of $50 \mathrm{~mm}$, and an aperture of $f / 22$. The specimen diameter corresponds to about 300 pixels. The frequency of image acquisition was set to 2 frames per second, and the correlation algorithm was performed with the VIC $3 \mathrm{D}{ }^{\circledR}$ commercial software. A Gaussian filter was applied to reduce noise. A facet size of 27 pixels with a grid spacing of 7 pixels was used. The 3-D DIC technique can calculate out-of-plane and in-plane full-field displacements of the cladding throughout the test. 


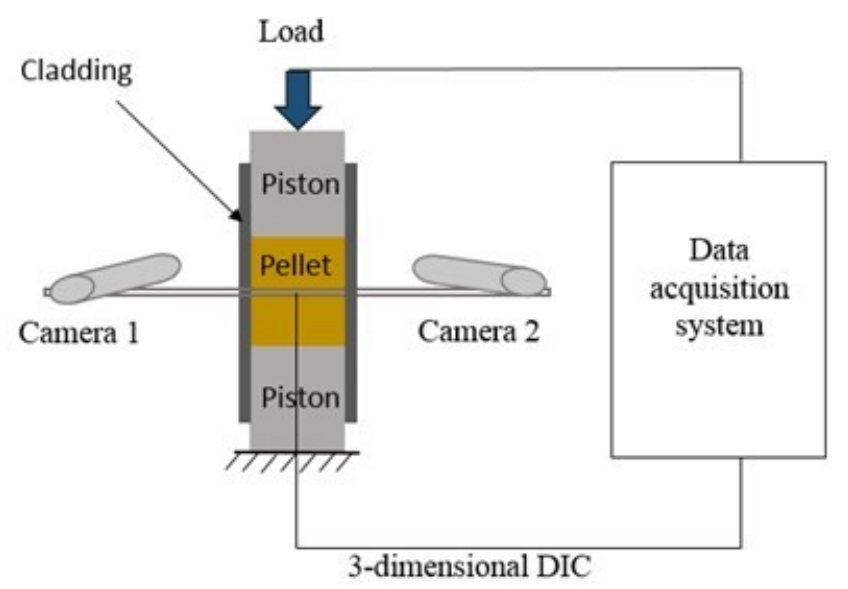

Fig. 3. Schematic representation of the EDC test setup and the 3D DIC system

Fig. 4 shows the hoop and axial Green-Lagrange strain components in the tube measured by the 3D DIC system. The strain at failure is recorded in the area of interest of the specimen, at point A in Fig. 4, where the axial and hoop strains reach their maximum absolute values. The axial strain gradient is high due to bulging, but the hoop strain gradient is relatively small, which allows the failure hoop strain to be determined reliably. The Green-Lagrange strains calculated by VIC-3D are transformed into logarithmic strains, which will be used throughout the remainder of this paper.

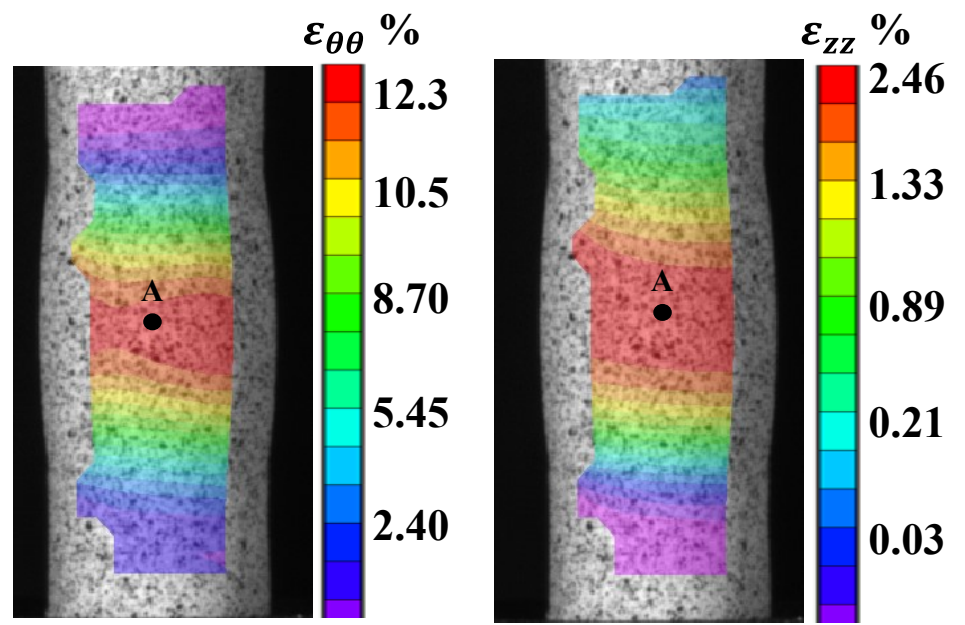

Fig. 4. Measured hoop and axial Green-Lagrange strain fields for an EDCT test

\section{Experimental results}

\subsection{Mechanical tests}

Two specimen shapes were used in this study. The first one has the thickness of industrial fuel cladding $(0.57 \mathrm{~mm})$. With this configuration, a strain ratio of 0.4 was achieved. Beyond this value, the excessive axial force causes the sample to rupture in tension outside of the area of interest. To reach higher biaxiality values, the thickness of the tube was reduced using a lathe to $0.52 \mathrm{~mm}$ (91\% of the initial thickness) over the central $14 \mathrm{~mm}$ of the sample. This solution leads to rupture in the area of interest at greater biaxiality values. It was verified that for a fixed-end EDC test and an EDCT test with a biaxiality equal to 0.21 , machining to this thickness did not affect the failure strain. The various EDCT tests performed with a wall thickness of $0.52 \mathrm{~mm}$ are listed in Table 1 . 


\begin{tabular}{|c|c|c|c|c|c|c|c|c|c|c|c|c|}
\hline $\begin{array}{c}\text { Biaxiality } \\
\varepsilon_{z z} / \varepsilon_{\theta \theta}\end{array}$ & -0.20 & -0.17 & -0.13 & 0.01 & 0.04 & 0.08 & 0.15 & 0.21 & 0.38 & 0.44 & 0.61 & 0.75 \\
\hline $\begin{array}{c}\varepsilon_{\theta \theta} \\
\text { at rupture }\end{array}$ & 0.316 & 0.274 & 0.222 & 0.123 & 0.095 & 0.102 & 0.113 & 0.115 & 0.116 & 0.120 & 0.127 & 0.137 \\
\hline
\end{tabular}

Table 1. Measured hoop strain at fracture of Zircaloy-4 tubes of thickness $0.52 \mathrm{~mm}$ for different strain biaxiality ratios

Fig. 5 presents the evolution of the hoop strain obtained from free-end, fixed-end, and EDCT tests as a function of strain biaxiality. All of the strain measurements correspond to point A, shown in Fig. 4. The last point of each curve corresponds to the rupture of the specimen, with the exception of the free-end EDC test, which reached a hoop strain of 0.53 without rupturing. The loading path of the free-end EDC test (black curve) is close to pure tension. Due to plastic anisotropy, the theoretical biaxiality in pure tension is not equal to -0.5 . At the beginning of this test, the strain ratio is close to -0.6 , and it increases to -0.3 at a hoop strain of 0.53. For the fixed-end EDC test (green curve), the strain ratio is initially -0.3 , and it increases to -0.07 at a hoop strain of 0.17 . Although the ends of this specimen were fixed, an axial shrinking was measured in the area of interest, and a positive axial strain was measured close to the ends of the sample.

In order to characterize the material ductility at low biaxiality, several EDCT tests (red curves) were performed at strain ratios less than zero. During these tests, the tie rods had a small cross section and allowed the specimen to shrink in the axial direction, but not as much as in the free-end EDC test. Using this design, rupture was observed at a hoop strain of 0.316 for a biaxiality of -0.20 . It was not possible to obtain a lower biaxiality in this study, because the tie rods would need to be thinner than could be machined with a uniform diameter. The minimum observed failure strain was 0.095 for a biaxiality of 0.04 , which is close to plane strain. As the biaxiality increased from 0.04 to 0.75 , the failure strain increased from 0.095 to 0.137 .

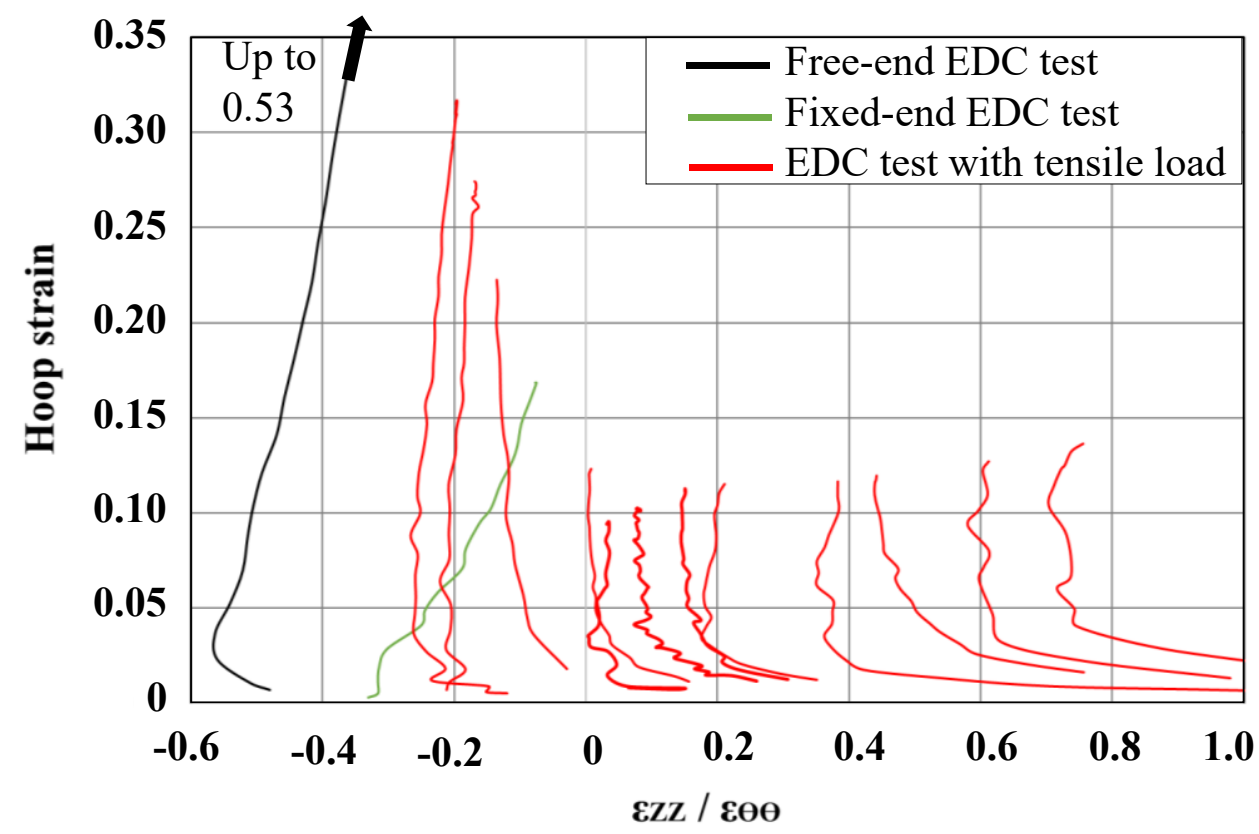

Fig. 5. Evolution of strain ratio for free-end, fixed-end, and EDCT tests

These same data are presented in a different manner in Fig. 6, in which the hoop strain is plotted versus the axial strain. Using this format, the data can be compared to Forming Limit 
Diagrams (FLD) that have been reported in the literature. The figure exhibits a characteristic $\mathrm{V}$-shape with a strongly decreasing ductility for biaxialities less than 0 and a slightly increasing ductility for biaxialities between 0 and 0.75 . This type of curve was found by [20] for AA6111-T4 aluminum sheets, and by [21] for aluminum 2008-T4 sheets using the hemispherical punch stretching method. FLDs were obtained for aluminum tubes in [22] and [23]. In [22], the tubes were loaded using a combination of internal pressure and axial force to vary the biaxiality. The authors observed a marked increase in ductility as the biaxiality increased from 0.1 to 1 . In [23], EDC tests were performed using rubber as the compression medium. The biaxiality was varied using a clamping system to decrease the effective free length of the specimen, which leads to an increase in biaxiality. The FLD at necking obtained by the authors exhibits the characteristic V-shape. The authors also measured the local strain at fracture by measuring the thickness of the specimen at the crack. It is interesting to note that this local strain at fracture decreases as the biaxiality increases up to 0.35 , but this trend should not be directly compared to the macroscopic behavior represented in Fig. 6. In the case of zirconium alloys, Yunchang and Koss [2] measured a decrease in the principal strain at failure when biaxiality increases from plain strain to equi-biaxial strain for hydrided Zircaloy-2 sheets using hemispherical punch tests, and they noted that void nucleation is strongly influenced by the presence of the hydrides. FLDs obtained from punch tests on Zirlo and Zircaloy-4 sheets [3-4] exhibit a V-shape similar to that of Fig. 6. Thus, the dependence of the failure strain on the biaxiality observed in this study is similar to trends exhibited by FLDs reported by several previous researchers.

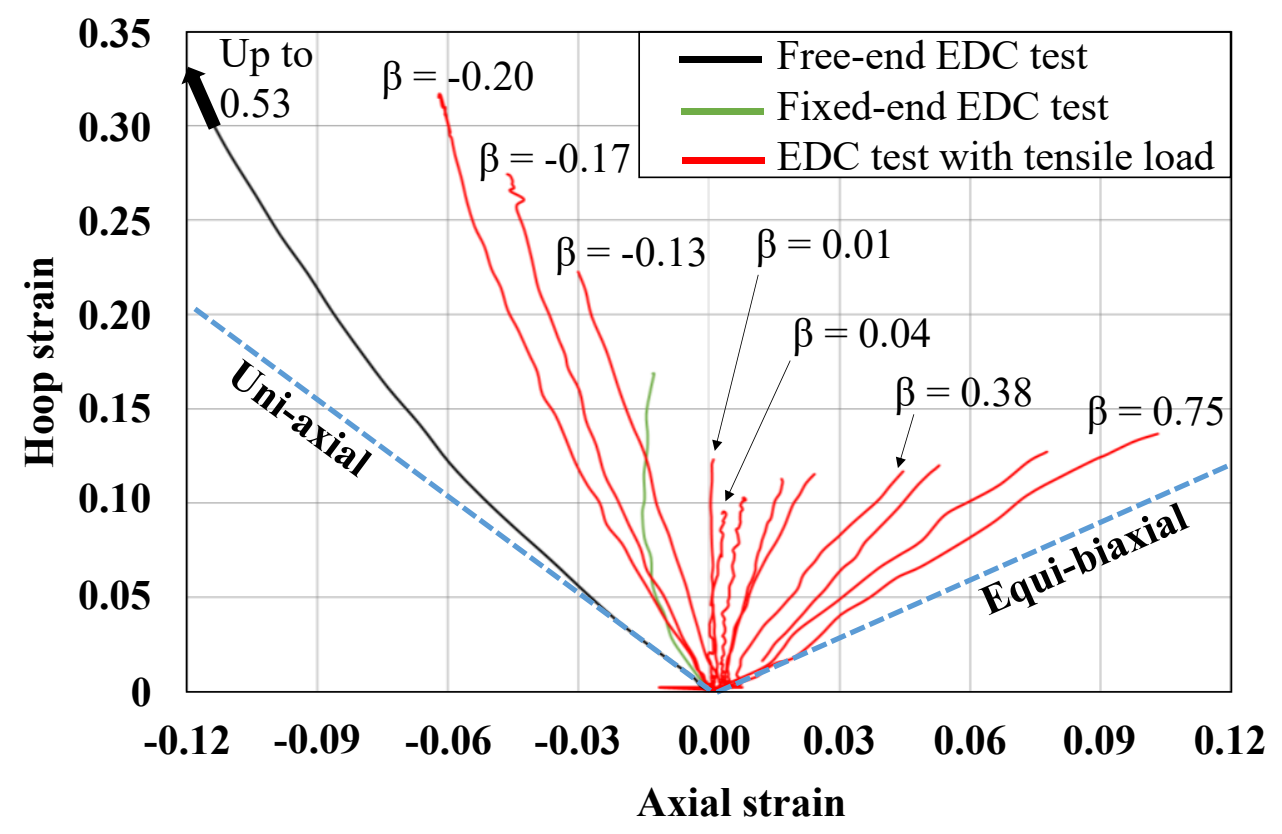

Fig. 6. Major (hoop) strain as a function of the minor (axial) strain.

\subsection{Observation of damage}

In the EDCT tests, failure occurs by axial propagation of a through-thickness crack, whose axial length at the end of the test is equal to the height of the media. The though-thickness crack initiates in the middle of the specimen, where the hoop strain is greatest, and propagates through the thickness of the sample at an angle close to $45^{\circ}$ to the principal direction of loading (the hoop direction). This failure scenario closely resembles the macroscopic failure mechanism observed during RIA experimental reactor tests [24-25]. 
The fracture surfaces of three of the EDCT tests were observed with a Scanning Electron Microscope (SEM). The samples were subjected to biaxialities, $\varepsilon_{z z} / \varepsilon_{\theta \theta}$, of 0.75 (Fig. 7a), 0.04 (Fig. 7b) and 0.20 (Fig. 7c). The fracture surfaces consist of very fine dimples (between 0.5 and $3 \mu \mathrm{m}$ ) that initiated at $\mathrm{Zr}(\mathrm{Fe}, \mathrm{Cr})_{2}$ strengthening particles (Laves phase) [26], some of which are circled in Fig. 7d. The dimples have approximately the same size for all biaxialities. However, the dimples for tests in which the absolute value of the biaxiality is large ( -0.20 and 0.75$)$ appear deeper, whereas they appear to be sheared for a biaxiality close to 0 . It therefore can be concluded that failure of Zircaloy-4 cladding is essentially controlled by void nucleation at fine strengthening particles followed by rapid coalescence by internal necking at high biaxiality [27] and possible coalescence by void shearing at low biaxiality [28].

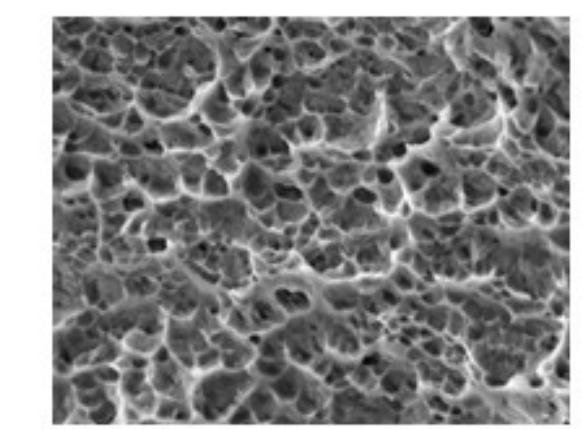

a)

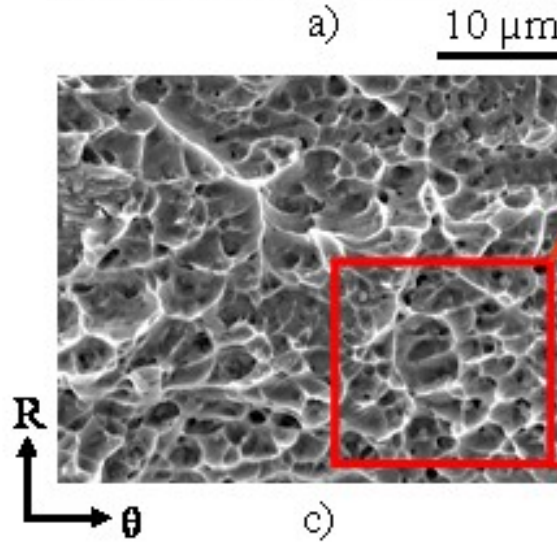

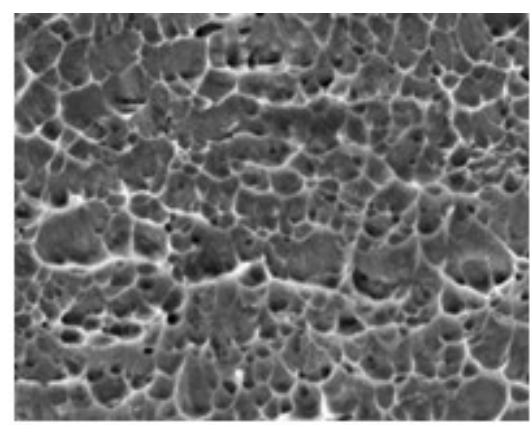

b)

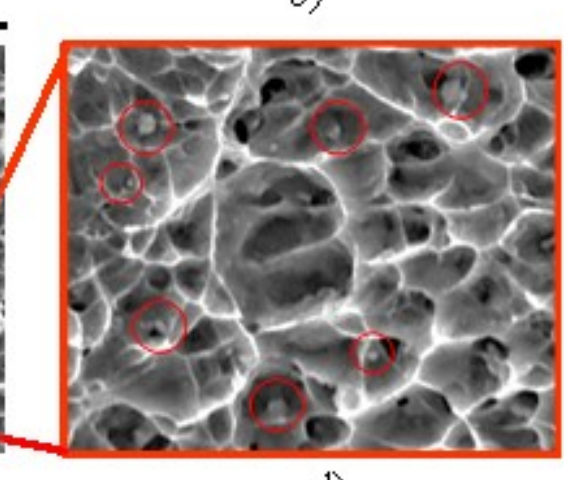

d)

Fig. 7 (a) Fracture surfaces observed for EDCT tests with biaxiality $\varepsilon_{z z} / \varepsilon_{\theta \theta}=0.75$, $\varepsilon_{\theta \theta}($ rupture $)=0.137$, (b) with biaxiality $\varepsilon_{z z} / \varepsilon_{\theta \theta}=0.04, \varepsilon_{\theta \theta}$ (rupture $)=0.095$, (c) with biaxiality $\varepsilon_{z z} / \varepsilon_{\theta \theta}=-0.20, \varepsilon_{\theta \theta}$ (rupture $)=0.316$ and $(d)$ Laves phase precipitates.

\section{Plastic behavior and modeling of the tests}

\subsection{Finite Element Analysis}

Finite element simulations were carried out to provide insight into the deformation and the failure modes observed during the tests. The simulations were performed using the Cast $3 \mathrm{M}$ software, which was developed by the CEA [29]. Quadrangular quadratic finite elements with 8 nodes and 4 Gauss points are used for the calculations, which are based on a large displacement and large rotation formulation. The geometry used for the finite element simulations of the EDCT tests is shown in Fig. 8, along with the boundary conditions. In order to simplify the calculations, only a half of the pellet and the cladding was modeled, as was done by previous researchers [9-11]. The left edge of the model is the axis of symmetry and is constrained in the radial (horizontal) direction. The bottom edge of the model is a plane of 
symmetry at the mid-plane of the sample and is constrained in the axial (vertical) direction. The tie rods are modeled as a single cylindrical structure with an appropriate cross-sectional area. The bottom surface of the tie rod is fixed to the upper surface of the fuel cladding. During the simulation, an axial (vertical) displacement is applied to the upper surface of the tie rod. A displacement of equal magnitude but opposite direction is applied to the upper surface of the piston that compresses the pellet. Both the tie rod and the fuel cladding experience axial tensile stress as a result of this load, and at the same time, the pellet expands as it is compressed to apply a tensile hoop strain to the cladding.

The simulations of the fixed-end and free-end EDC tests use this same geometry, but without the tie rods. For the free-end EDC simulations, the top surface of the cladding tube is free and unconstrained. For the fixed-end EDC simulations, the top surface of the cladding tube is constrained in the axial (vertical) direction.

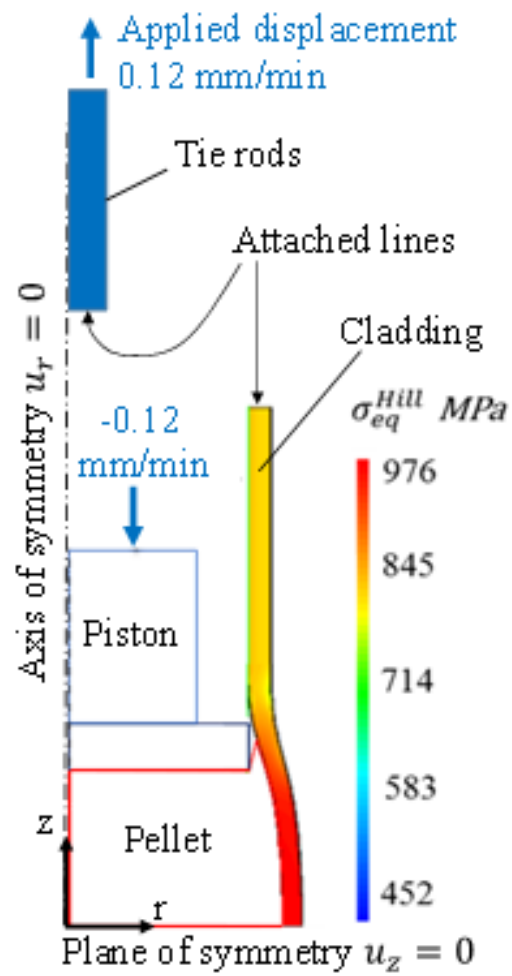

Fig. 8. Geometry and boundary conditions used for the finite element simulations of the EDCT tests

During the tests, frictional contact occurs between the piston and the pellet, and between the pellet and the cladding. The friction is modeled by Coulomb's friction law in the Cast $3 \mathrm{~m}$ software. It is not possible to experimentally measure the coefficients of friction between the different components. Therefore, simulations were used to identify suitable values.

A series of simulations revealed that the coefficient of friction between the pistons and the pellet has negligible influence on the simulated force-displacement response and on the local deformation of the specimen. Therefore, to facilitate the simulations, a coefficient of 0 was used for this interface. However, the coefficient of friction between the pellet and the cladding has a significant effect on the simulated strain field, strain biaxiality, and forcedisplacement response. Values of $0.1,0.2$, and 0.4 were used in the simulations for this interface. A coefficient of friction of 0.4 leads to the closest agreement between the simulations and the experimental results for all types of EDC tests considered. Therefore, this value was 
used for all of the simulations in this study. It is interesting to note that Desquines et al [30] also used a value of 0.4 for the coefficient of friction between the pellet and the cladding in their simulations.

\subsection{Material behavior}

To simplify the model describing the behavior of the polymer pellet, the effect of the strain rate is not accounted for. Because polymers exhibit a strong strain rate dependence, the model parameters given below should only be used for the strain rate used in this study $\left(10^{-4} \mathrm{~s}^{-1}\right)$. In the simulations, the flow stress of the polymer pellet, $\bar{\sigma}_{\text {pel }}$ (based on von Mises plasticity), is expressed as:

$$
\bar{\sigma}_{\mathrm{pel}}=\sigma_{y}+K \bar{\epsilon}_{p}^{n}
$$

$\bar{\epsilon}_{p}$ is the equivalent plastic strain, $\sigma_{y}$ is the yield strength equal to $8 \mathrm{MPa}, K$ is the strength coefficient equal to $30 \mathrm{MPa}$, and $n$ is the strain hardening exponent equal to 0.5 . Young's modulus is $0.6 \mathrm{GPa}$ and Poisson's ratio is 0.47 .

A unified anisotropic viscoplastic model for the cladding tube, which was specifically identified in RIA-PCMI conditions by Le Saux et al. [31], was used in this work. The complete model accounts for strain hardening, temperature, strain rate and irradiation conditions. A simplified version is used in this work. The plastic orthotropy of the material is described by a Hill quadratic yield criterion [32] with isotropic hardening. The equivalent stress $\sigma_{H}$ is given in the orthotropic radial-circumferential-axial $(r, \theta, z)$ coordinate system of the tube as:

$$
\begin{gathered}
\sigma_{H}=\left[H_{r r}\left(\sigma_{\theta \theta}-\sigma_{z z}\right)^{2}+H_{\theta \theta}\left(\sigma_{z z}-\sigma_{r r}\right)^{2}+H_{z z}\left(\sigma_{r r}-\sigma_{\theta \theta}\right)^{2}+2 H_{r \theta} \cdot \sigma_{r \theta}^{2}\right. \\
\left.+2 H_{r z} \cdot \sigma_{r z}^{2}+2 H_{\theta z} \cdot \sigma_{\theta z}^{2}\right]^{1 / 2}
\end{gathered}
$$

The following coefficients are used following Le Saux et al. [31] :

$$
H_{r r}=0.58 \quad H_{\theta \theta}=0.42 \quad H_{z z}=0.41 \quad H_{r \theta}=H_{r z}=H_{\theta z}=1.5
$$

The yield surface is expressed as: $\phi=\sigma_{H}-\bar{\sigma}_{\mathrm{zir}}=0$ where $\bar{\sigma}_{\mathrm{zir}}$ is the flow stress of the cladding material. The plastic strain rate tensor is calculated by the normality rule and is given by:

$$
\underline{\dot{\varepsilon}}_{p}=\dot{p} \frac{\partial \sigma_{H}}{\partial \underline{\sigma}}
$$

$p$ is the equivalent strain associated with the Hill criterion. $\bar{\sigma}_{\text {zir }}$ is plotted as a function of $p$ in Fig. 9. Young's modulus and Poisson's ratio for zirconium are $E=95 \mathrm{GPa}$ and $v=$ 0.34. Tensile tests were performed to identify the behavior of the $304 \mathrm{~L}$ steel used for the tie rods, as shown in Fig. 9. Von Mises plasticity is used to describe its behavior. Young's modulus and Poisson's ratio for 304L steel are taken as: $E=200 \mathrm{GPa}$ and $v=0.3$. Finite element simulations were then used to select the cross section and the length of the tie rods to obtain the desired strain biaxiality in the sample during the EDCT tests. 


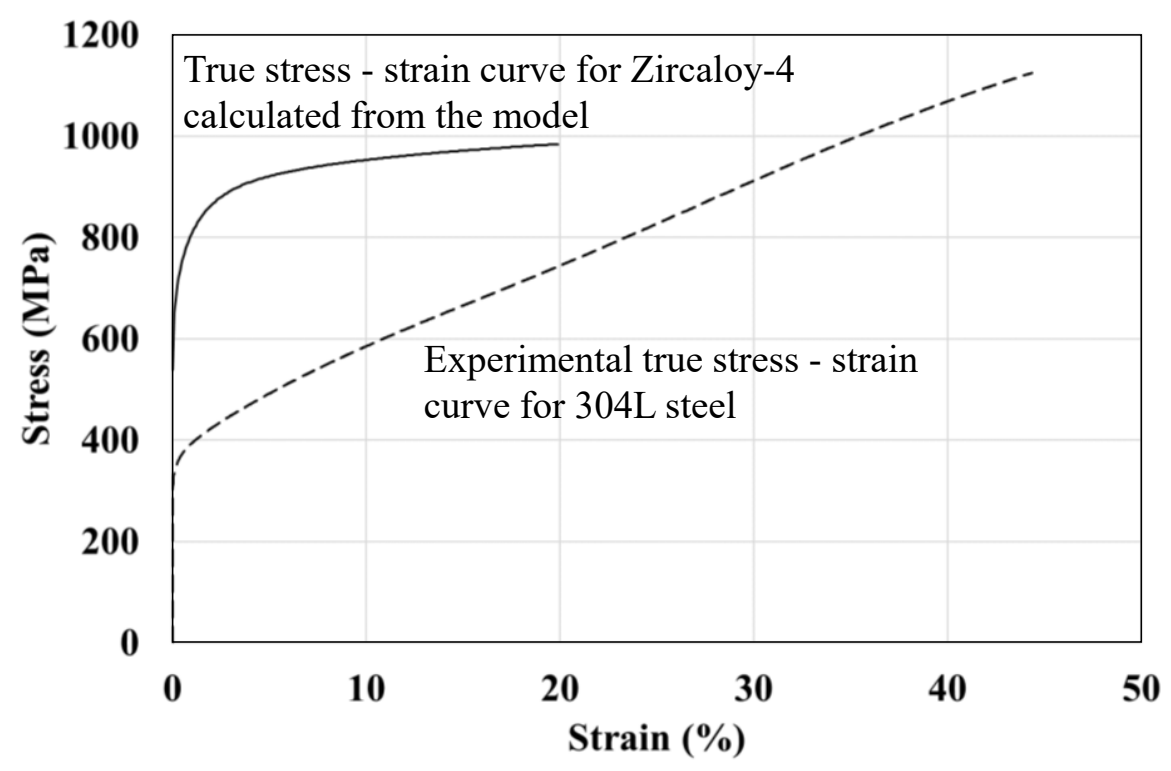

Fig. 9. True stress-strain curves for 304L steel and Zircaloy-4

The simulated hoop strain versus biaxiality curves are compared with the experimental data in Fig. 10. The simulations are in good agreement with the tests, and the different levels of biaxiality are well reproduced. At the beginning of each of the EDCT tests, the biaxiality is very high due to the fact that the tie rods start pulling on the cladding before the media begins to impose a hoop strain. The biaxiality then settles to its final value at a hoop strain of a few percent. The fixed-end EDC test (dark green symbols and curve) is also well reproduced by the simulation. For the cases where the biaxiality is less than 0 , the pellet becomes severely deformed and tends to flow between the piston and the cladding. This large distortion leads to convergence difficulties, and the simulations terminate before reaching the rupture point. These simulations are indicated with a star marking the last computed step before the simulation diverged. Each of the simulations can then be post-processed to create a rupture initiation criterion, as discussed in the following section.

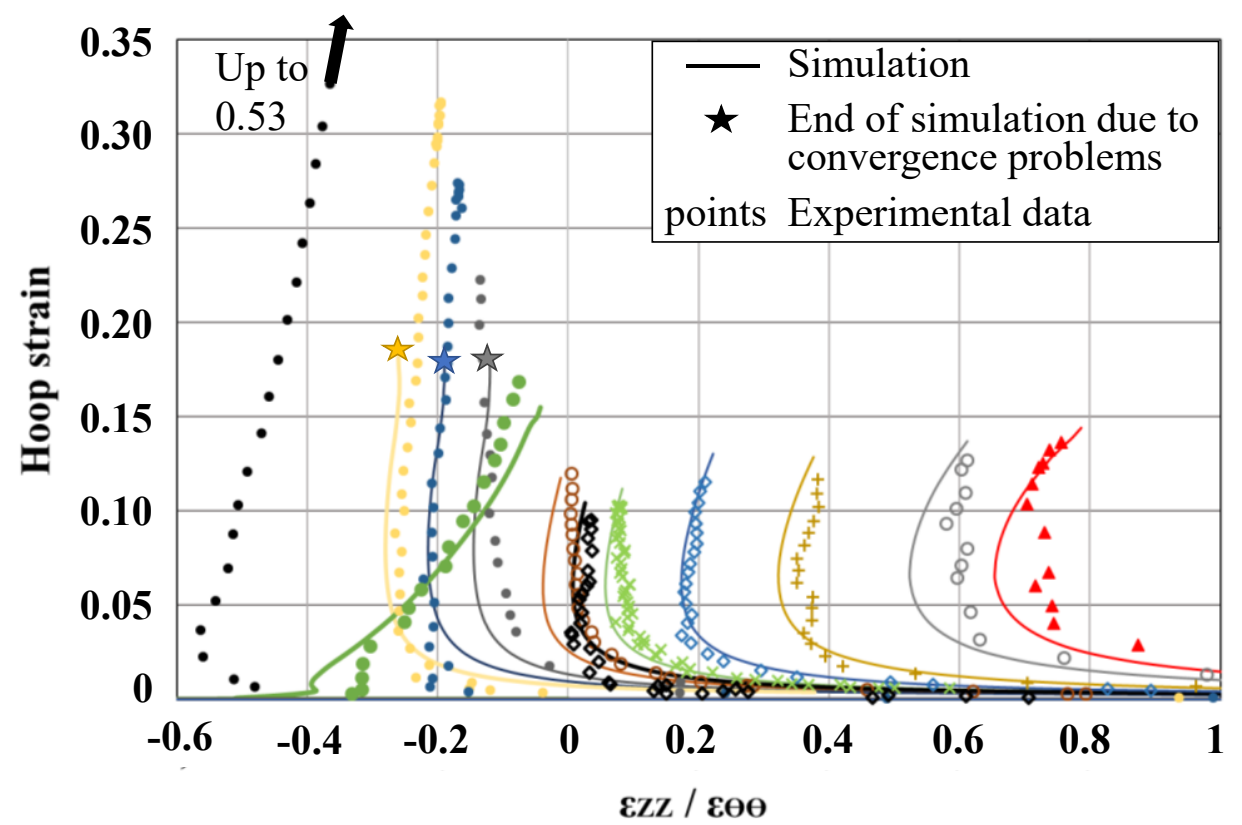

Fig. 10. Comparison of the simulated loading paths (solid lines) and experimental data (points) at the mid-plane of the external surface of the sample 


\section{Damage model}

\subsection{Model}

Ductile fracture is often described by three distinct phases [33]. In the first phase, cavities nucleate on defects (Laves phase in the case of Zircaloy-4). If the stress triaxiality is high, the cavities can then grow and eventually coalesce to form a macroscopic crack (Fig. 7a and Fig. 7c). Therefore, many micromechanical models of void growth and coalescence [3435] consider stress triaxiality as the driving force for damage and rupture. Unfortunately, these models are not very accurate for certain situations in which the stress triaxiality is low, which is the case for the thin cladding tubes used in this study. For example, these models predict that for shear loading, cavities do not increase in size and do not coalesce, which leads to unrealistically high predictions of the ductility. In shear loading, damage and void coalescence may occur via fine localization bands within the material, via changes in the shape and orientation of the cavities that can lead to coalescence [28], or via coalescence triggered by void sheeting [36-38] or void shearing [39-41], similar to that seen in Fig. 7b. In fact, researchers have observed that ductility in pure shear can be less than that in pure tension [42-43], despite the lower stress triaxiality. To be able to represent these experimental trends, shear effects can be considered by including the Lode parameter in the fracture model [42, 44, 45].

In this work, a macroscopic fracture initiation model is proposed to describe failure for all levels of biaxiality. The proposed model is "uncoupled" so that the effect of damage on the elasto-plastic behavior is neglected. Uncoupled models for ductile fracture are based on the definition of a damage indicator, $D$, which is computed by post-processing elasto-plastic finite element simulations. For each material point (Gauss points in the finite element simulations) $D$ is computed for each time step, $t$, as:

$$
D(t)=\int_{0}^{p(t)} W(\text { stress state }) d p
$$

Fracture is assumed to initiate when $D$ reaches 1 . The fracture strain $p_{f}$ is the value of the cumulated plastic strain at the fracture initiation point. However, due to the stress state dependence of the damage evolution law, the fracture strain $p_{f}$ does not necessarily correspond to the maximum value of cumulated strain in the entire structure. Note that for a constant stress state, $p_{f}=1 / W$.

Several forms for the $W$ function have been proposed in the literature. For high stress triaxialities, $W$ can take the form of the Rice \& Tracey model, in which void growth rate is affected only by the triaxiality, $W=f(T)=\alpha \exp (\beta T)$ [46] where $\alpha$ and $\beta$ are model parameters, and $T$ is the stress triaxiality ratio defined as:

$$
T=\frac{1}{3} \frac{\sigma_{k k}}{\sigma_{e q}}
$$

$\sigma_{e q}$ is the von Mises equivalent stress. In the case of plastically anisotropic materials, the use of "isotropic" quantities such as stress triaxiality or Lode parameter might not be relevant to describe failure [47]. In this case, it was recently proposed (based on unit cell simulations) to use a modified triaxiality ratio $\left(T^{*}\right)$ to describe damage growth [48]. It is defined as:

$$
T^{*}=\frac{1}{3} \frac{\sigma_{k k}}{\sigma_{H}}
$$


$\sigma_{H}$ is the Hill equivalent stress defined in eq. (2). In the case of moderate stress triaxiality, the $W$ damage function can be written as a function of both the stress triaxiality and the Lode parameter using an additive form, $W=f(T)+g(L)$ as proposed in [49] to represent failure of an ultra-high strength steel, or using a multiplicative form $W=f(T) \times g(L)$ proposed in [50] and [51] to represent failure of pipeline steels. More complex forms for $W$ have been proposed in the literature. Most of them are reviewed in [42]. In this work, the following definition of the Lode parameter will be used for any second order tensor $\underline{t}$. Let $\underline{t}^{\prime}$ be the deviator of $t$ and $t_{1}^{\prime} \geq t_{2}^{\prime} \geq t_{3}^{\prime}$ its eigenvalues. The Lode parameter of tensor $\underline{t}$ is then defined as $[49,52]$ :

$$
\operatorname{Lode}(\underline{t})=-3 \frac{t_{2}^{\prime}}{t_{1}^{\prime}-t_{3}^{\prime}}
$$

Note that the value of $\operatorname{Lode}(\underline{t})$ is between -1 and 1 . To define the $W$ function, the Lode parameter of the stress tensor is often used [42]. The Lode parameter is, however, used to describe the fact that ductility is minimum under generalized plane strain conditions, which can lead to easy band localization [53]. Based on this consideration, it is proposed in this work to express the failure criterion as a function of the Lode parameter of the plastic strain rate tensor $\dot{\varepsilon}_{p}$. Note that in the case of von Mises plasticity, the Lode parameter of the stress tensor ( $L$ in the following) is equal to that of the plastic strain rate tensor (referred to as $L^{*}$ in the following).

In this work, the thickness of the tube is such that a plane stress state is almost achieved. In this case, the Lode parameter and the triaxiality are linked. In the case of von Mises plasticity with a strain biaxiality between -0.5 and 1 , this relation is expressed as:

$$
T=\frac{(3-L)}{3 \sqrt{3+L^{2}}}
$$

In the case of Hill plasticity, the relation between $T^{*}$ and $L^{*}$ cannot be explicitly expressed. This relation is plotted in Fig. 11 for the investigated strain biaxiality range and the indicated Hill parameters.

In this study, the damage $W$ is expressed as a function of the Lode parameter $L^{*}$ as:

$$
W=a\left(1-\left|L^{*}\right|^{b}\right)+c\left(1-\operatorname{sign}\left(L^{*}\right)\left|L^{*}\right|^{d}\right)+W_{0}
$$

$a, b, c, d$ and $W_{0}$ are parameters to be adjusted. $\operatorname{sign}\left(L^{*}\right)$ is equal to -1 if $L^{*}<0$ and to +1 if $L^{*}>0$. Note that the function has an even part $\left(\propto\left|L^{*}\right|^{b}\right)$ and an odd part $\left(\propto \operatorname{sign}\left(L^{*}\right)\left|L^{*}\right|^{d}\right)$ to distinguish equibiaxial tension $(L=-1)$ and pure tension $(L=1)$. 


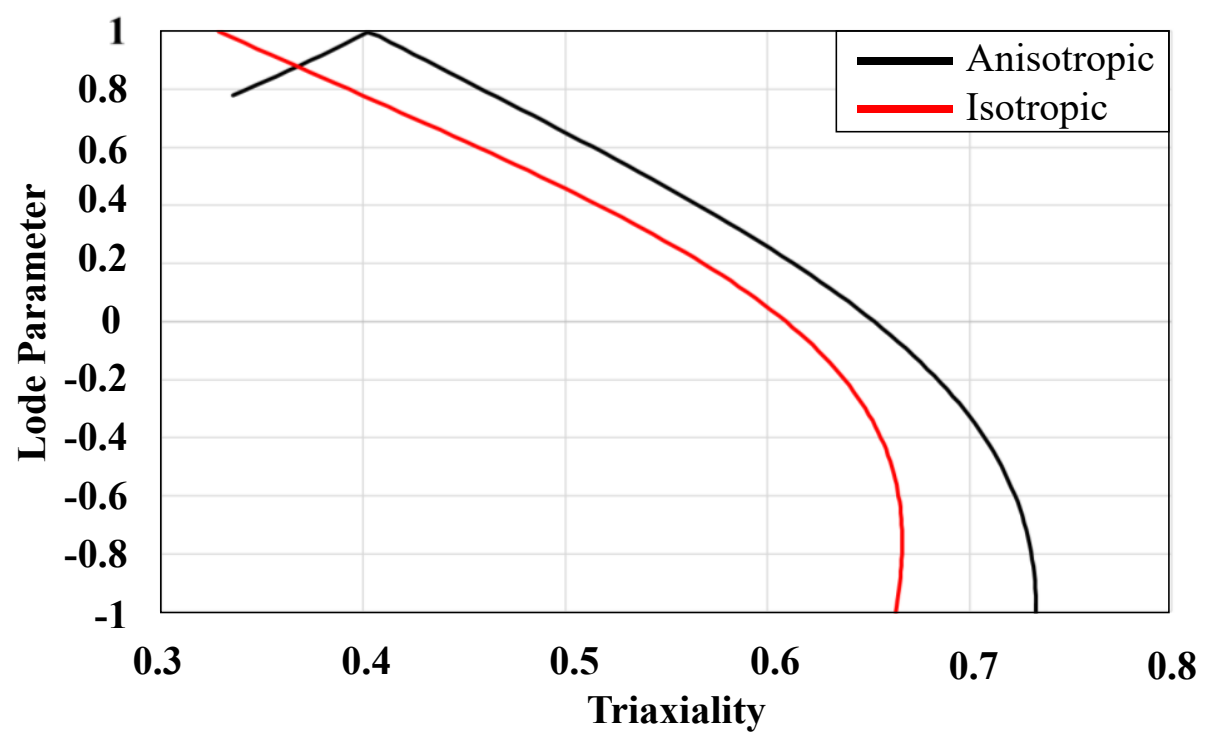

Fig. 11. Dependence between triaxiality and Lode parameter for a von Mises material (isotropic) and a Hill material (with the parameters used in this study).

An optimization software is used to tune the model parameters $\left(a, b, c, d, W_{0}\right)$ using the Levenberg-Marquardt algorithm [54]. Elasto-plastic simulations for several biaxiality levels were post-processed up to the experimental failure point (hoop strain at failure) as recorded by stereo-correlation. The maximum value of damage over specimen $i, D_{i}^{\max }$, is computed for this time step. The cost function is then defined as:

$$
\delta=\sum_{i=1}^{N}\left(D_{1}^{\max }-1\right)^{2}
$$

$D^{\max }=1$ corresponds to failure of the specimen. The $N$ specimens considered in the fit are only those for which $\beta>-0.1$. The optimized damage parameters are listed in Table 2 .

\begin{tabular}{|c|c|c|c|c|}
\hline $\mathrm{a}$ & $\mathrm{b}$ & $\mathrm{c}$ & $\mathrm{d}$ & $\mathrm{W}_{\mathrm{o}}$ \\
\hline 10.576 & 0.232 & 0.456 & 0.641 & 0.7 \\
\hline
\end{tabular}

Table 2. Parameters of the damage model

\subsection{Macroscopic failure point}

The predicted and experimental macroscopic failure points are compared in Fig. 12. For strain biaxialities between 0 and 0.75 , the failure point can be evaluated by post-processing finite element simulations. The agreement for these tests is very good. In particular, strain to failure is minimum for a biaxiality equal to 0 and slowly increases as biaxiality increases. Below 0 , it was not possible to carry out finite element simulations up to the experimental failure point, because the simulations encountered convergence problems at a hoop strain of approximately 0.18 . In these cases, the failure strain was calculated assuming a fixed strain biaxiality (red symbols). In practice, the simulations were carried out using a single element with a prescribed deformation gradient corresponding to the desired biaxiality. Nonetheless, a very good agreement is found when comparing the experimental and simulated strains to failure. 


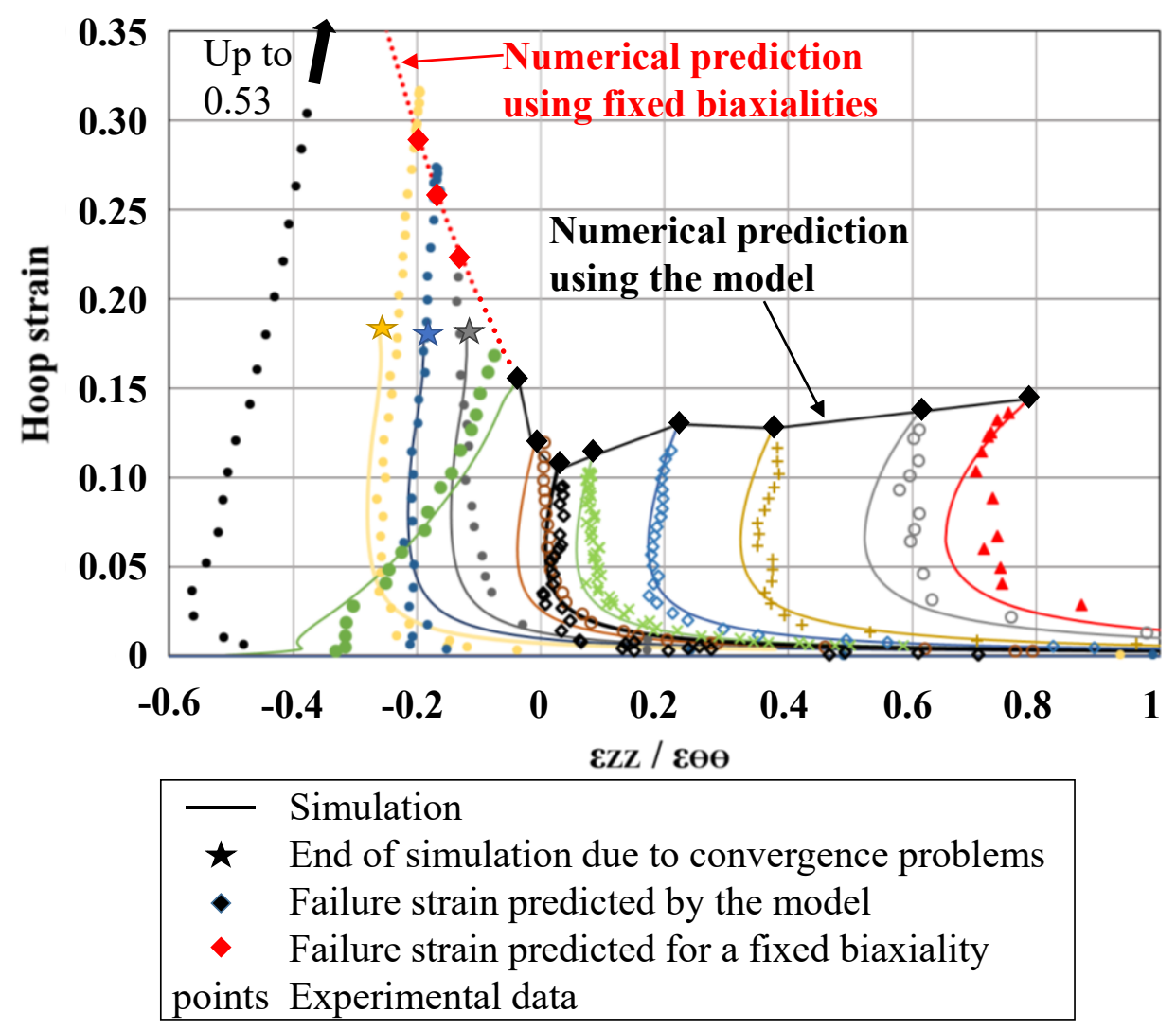

Fig. 12. Numerical predictions and experimental results

Contour plots of the damage indicator are presented in Fig. 13 for levels of biaxiality at failure equal to 0.04 and 0.75 . For both levels of strain biaxiality, damage was localized near the mid-plane of the cladding. For plane strain conditions (biaxiality of 0.04), fracture is predicted to initiate at the external wall of the cladding. At higher biaxiality, it initiates at the inner wall. As failure occurs very rapidly, these trends could not be verified experimentally. This result is the outcome of a complex load history. Regardless of the biaxiality, the maximum cumulated plastic strain is reached at the inner diameter, mainly because the maximum hoop strain is reached at this location. Friction and bending generate a Lode parameter gradient, which causes the initiation point to move from the outer diameter (at low biaxiality) to the inner diameter (at high biaxiality). 


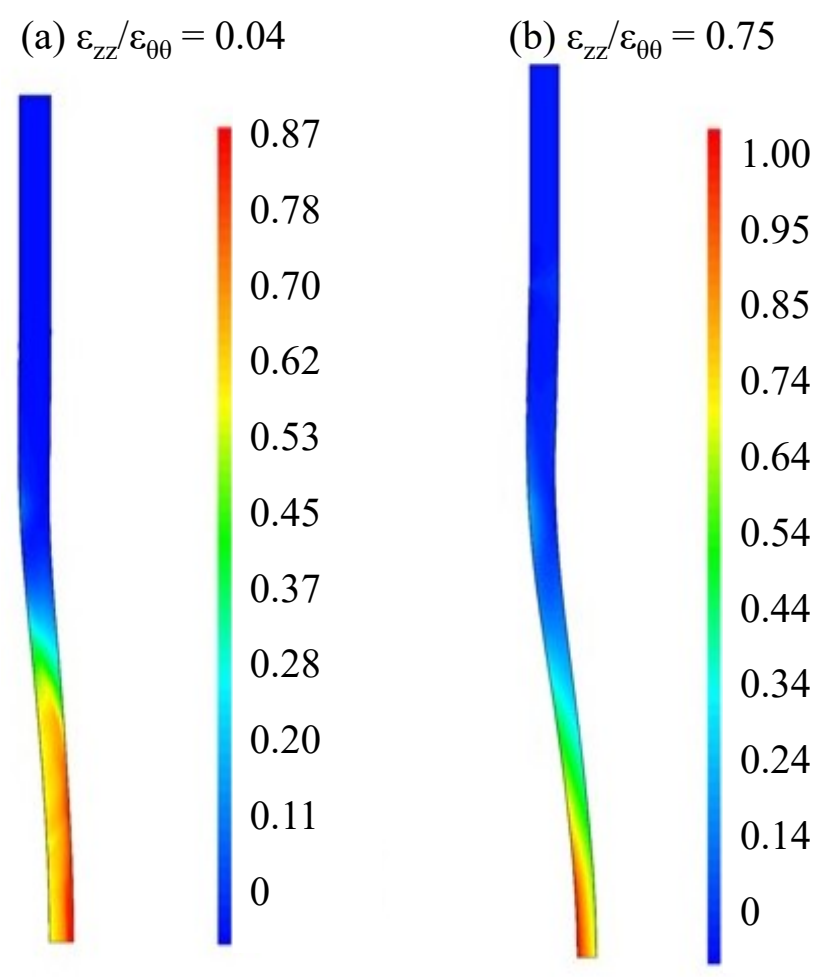

Fig. 13. Damage distribution computed for the experimental failure for (a) biaxiality $=0.04$ and (b) biaxiality $=0.75$

\section{Conclusions}

During a Reactivity Initiated Accident (RIA), nuclear fuel cladding experiences a multiaxial loading state in which the Pellet-Cladding Mechanical Interaction (PCMI) produces a strain biaxiality ratio $\varepsilon_{z z} / \varepsilon_{\theta \theta}$ of between 0 and 1 . The effect of loading path on the onset of ductile fracture of non-irradiated Cold-Worked Stress Relieved (CWSR) Zircaloy-4 fuel cladding is studied by submitting tubular specimens to strain states with biaxiality ratios between -0.20 and 0.75 . The results show that as the biaxiality ratio increases from -0.20 to 0.04 , the hoop strain at fracture decreases sharply from 0.316 to 0.095 . However, for biaxiality ratios between 0.04 and 0.75 , the hoop strain at fracture increases from 0.095 to 0.137 . Examination of fracture surfaces shows that failure is essentially controlled by the coalescence of very small voids that nucleate at strengthening particles. Rupture appears to occur by coalescence via void shearing at low biaxialities, and by coalescence via internal necking at high biaxialities. Simulations using elasto-plastic behaviors of the cladding, pellets and tie rods are used to determine the loading path to fracture for all biaxiality levels. A ductile failure initiation model is proposed, based on the Lode parameter of the plastic strain rate tensor to account for the changes in failure mechanisms and strains to failure for the different levels of biaxiality. The model shows very good agreement between experimental and simulated strains at failure.

\section{Acknowledgements}

The authors would like to thank the project GAINE of the French Institut Tripartite CEA-EDF-Framatome for supporting this study. 


\section{References}

[1] J. Papin, B. Cazalis, J. M. Frizonnet, J. Desquines, F. Lemoine, V. Georgenthum, F. Lamare and M. Petit, "Summary and Interpretation of the CABRI REP-Na Program," Nuclear Technology, no. 1, pp. 230-250, 2017.

[2] F. Yunchang and D. Koss, "The Influence of Multiaxial States of Stress on the Hydrogen Embrittlement of Zirconium Alloy Sheet," Metallurgical Transactions A 16, p. $675-681,1985$.

[3] Y. Seo, H. C. Hum, H. Lee and N. Kim, "Forming Limit Diagrams of Zircaloy-4 and Zirlo Sheets for Stamping of Spacer Grids of Nuclear Fuel Rods," The Korean Society of Mechanical Engineers, pp. 889-897, 2011.

[4] M. Kim, F. Rickhey, H. Lee and N. Kim, "Analytical determination of forming limit curve for zirlo and its experimental validation," Journal of Manufacturing Processes, vol. 23, pp. 122-129, 2016.

[5] E. Kaplar, L. Yegorova, K. Lioutov, A. Konobeyev and N. Jouravkova, "Mechanical Properties of Unirradiated and Irradiated $\mathrm{Zr}-1 \% \mathrm{Nb}$ Cladding," International Agreement report, 2001.

[6] M. K. Khan and M. Pathak, "Ballooning Deformation of Zircaloy-4 Fuel Sheath," Journal of Pressure Vessel Technology, vol. 136, pp. 031206-12, 2014.

[7] Y. Mishima, "An Improved Testing Device for Measuring Tangential Expandability of Beryllium Tube," Journal of Nuclear Science and Technology, pp. 294-297, 1966.

[8] A. Garlick, "Fracture of zircaloy cladding under simulated power ramp conditions," Journal of nuclear materials, pp. 209-224, 1973.

[9] V. Grigoriev, R. Jakobsson and D. Schrire, "Experimental Evaluation of Critical Strain Energy Density for Irradiated Cladding under Simulated RIA Condition," ENS Topfuel Stockholm, Sweden, 2001.

[10] O. Dufourneaud, A. G. Varias, V. Grigoriev, R. Jakobsson and D. Schrire, "Numerical simulation of the expansion due to compression test," Fuel Safety Research Specialists Meeting, Tokai, Japan, 2001.

[11] O. Dufourneaud and A. G. Varias, "Elastic-Plastic Deformation of a Nuclear Fuel Cladding Specimen under the Internal Pressure of a Polymer Pellet," Computational Mechanics Congress, Vienna, Austria, 2002.

[12] Y. Ménager and A. Varias, "Effect of External Hydride Layer on Fuel-Cladding Deformation during EDC-Testing," 17th International Conference on Structural Mechanics in Reactor Technology, 2003.

[13] M. Le Saux, C. Poussard, X. Averty, C. Sainte Catherine, S. Carassou and J. Besson, "High Temperature Expansion Due to Compression Test for the Determination of a Cladding Material Failure," International LWR Fuel Performance Meeting, San Francisco, California, 2007.

[14] F. Latourte, N. Rupin, M. He, A. Parrot and S. Leclercq, "Full field measurements used for assessing industrial issues -Two examples," Full field measurements and identification in Solid Mechanics, vol. 4, pp. 92-105, 2012. 
[15] B. N. Nobrega, J. S. King and G. S. Was, "Improvements in the design and analysis of the segmented expanding mandrel test," Journal of Nuclear Materials, vol. 131, pp. 99104, 1985.

[16] A. Hellouin de Menibus, Q. Auzoux, P. Mongabure, V. Macdonald, T. Le Jolu, J. Besson and J. Crépin, "Fracture of Zircaloy-4 cladding tubes with or without hydride blisters in uniaxial to plane strain conditions with standard and optimized expansion due to compression tests," Materials Science and Engineering: A, vol. 604, pp. 57-66, 2014.

[17] T. Shinozaki, T. Mihara, Y. Udagawa, T. Sugiyama and M. Amaya, "Failure Behavior of the Cladding with Outer Surface Pre-crack in Biaxial Stress Test," Proceedings WRFPM , pp. 14-17, 2014.

[18] T. Shinozaki, Y. Udagawa, T. Mihara, T. Sugiyama and M. Amaya, "Improved-EDC tests on the Zircaloy- $\square$ cladding tube with an outer surface pre-crack," Journal of Nuclear Science and Technology, vol. 53, pp. 1426-1434, 2016.

[19] F. Li, T. Mihara, Y. Udagawa and M. Amaya, "The effect of hydride morphology on the failure strain of stress-relieved Zircaloy-4 cladding with an outer surface pre-crack under biaxial stress states," Journal of Nuclear Science and Technology, pp. 432-439, 2019.

[20] M. Jain, J. Allin and D. J. Lloyd, "Fracture limit prediction using ductile fracture criteria for forming of an automotive aluminum sheet," International Journal of Mechanical Sciences, vol. 41, pp. 1373-1288, 1999.

[21] A. Graf and W. Hosforf, "Effect of Changing Strain Paths on Forming Limit Diagrams of AI 2008-T4," Metallurgical Transactions A volume, vol. 24, 1993.

[22] Z. He, Z. Wang, Y. Lin, H. Zhu and S. Yuan, "A modified Marciniak-Kuczynski model for determining the forming limit of thin-walled tube extruded with initial eccentricity," International Journal of Mechanical Sciences, vol. 151, pp. 715-723, 2019.

[23] J. P. Magrinho, M. B. Silva, G. Centeno, F. Moedas, C. Vallellano and P. A. F. Martins, "On the determination of forming limits in thin-walled tubes," International Journal of Mechanical Sciences, vol. 155, pp. 381-391, 2019.

[24] NEA, "Nuclear Fuel Behaviour Under Reactivity-initiated Accident (RIA) Conditions," Nuclear Energy Agency, 2010.

[25] T. Fuketa, F. Nagase, K. Ishijima and T. Fujishiro, "NSRR/RIA experiments with highburnup PWR fuels.," Nuclear Safety, vol. 37, no. 328-342, 1996.

[26] M. Le Saux, J. Besson, S. Carassou, C. Poussard and X. Averty, "Behavior and failure of uniformly hydrided Zircaloy-4 fuel claddings between $25^{\circ} \mathrm{C}$ and $480{ }^{\circ} \mathrm{C}$ under various stress states, including RIA loading conditions," Engineering Failure Analysis, vol. 17, pp. 683-700, 2010.

[27] P. F. Thomason, "A three-dimensional model for ductile fracture by the growth and coalescence of microvoids," Acta Metallurgica, vol. 33, pp. 1087-1095, 1985.

[28] M. E. Torki and A. A. Benzerga, "A mechanism of failure in shear bands," Extreme Mechanics Letters, vol. 23, pp. 67-71, 2018.

[29] http///www-cast3m.cea.fr. 
[30] J. Desquines, D. A. Koss, A. T. Motta, B. Cazalis and M. Petit, "The issue of stress state during mechanical tests to assess cladding performance during a reactivity-initiated accident (RIA)," Journal of Nuclear Materials, vol. 412, pp. 250-267, 2011.

[31] M. Le Saux, J. Besson, S. Carassou, C. Poussard and X. Averty, "A model to describe the anisotropic viscoplastic mechanical behavior of fresh and irradiated Zircaloy-4 fuel claddings under RIA loading conditions," Journal of Nuclear Materials, vol. 378, pp. 60-69, 2008.

[32] R. Hill, "The Mathematical Theory of Plasticity," Clarendon Press, Oxford., 1950.

[33] W. M. Garrison and N. R. Moody, "Ductile fracture," Journal of Physics and Chemistry of Solids, pp. 1035-1074, 1987.

[34] F. M. Beremin, "Cavity formation from inclusions in ductile fracture of A508 steel," Metal Trans A, p. 723-731, 1981.

[35] G. Le Roy, J. D. Embury, G. Edwards and M. F. Ashby, "A model of ductile fracture based on the nucleation and growth of voids," Acta Metall, vol. 29, pp. 1509-1522, 1981.

[36] M. J. Haynes and R. P. Gangloff, "Temperature-dependent void-sheet fracture in Al-CuMg-Ag-Zr," Metallurgical and Materials Transactions A, pp. 1599-1613, 1998.

[37] B. Tanguy, T. T. Luu, G. Perrin, A. Pineau and J. Besson, "Plastic and damage behavior of a high strength X100 pipeline steel : experiments and modelling," International Journal of Pressure Vessels and Piping, vol. 85, pp. 322-335, 2008.

[38] F. Bron, J. Besson and A. Pineau, "Ductile rupture in thin sheets of two grades of 2024 aluminum alloy," Materials Science and Engineering: A, vol. 380, pp. 356-364, 2004.

[39] H. Rogers, "The tensile fracture of ductile metals," AIME Trans, vol. 218, pp. 498-506, 1960.

[40] A. Pineau, A. A. Benzerga and T. Pardoen, "Failure of metals I: Brittle and ductile fracture," Acta Materialia, vol. 107, pp. 424-483, 2016.

[41] P. J. Noell, D. J. Carroll and L. Boyce, "The mechanisms of ductile rupture," Acta Materialia, vol. 161, pp. 83-98, 2018.

[42] Y. Bai and T. Wierzbicki, "A new model of metal plasticity and fracture with pressure and Lode dependence," International Journal of Plasticity, vol. 24, pp. 1071-1096, 2008.

[43] Y. Bai and T. Wierzbicki, "A comparative study of three groups of ductile fracture loci in the 3D space," Engineering Fracture Mechanics, vol. 135, pp. 147-167, 2015.

[44] M. Dunand and D. Mohr, "On the predictive capabilities of the shear modified Gurson and the modified Mohr-Coulomb fracture models over a wide range of stress triaxialities and lode angles," Journal of the Mechanics and Physics of Solids, vol. 59, pp. 1374-1394, 2011.

[45] T. S. Cao, J. M. Gachet, P. Montmitonnet and P. O. Bouchard, "A Lode-dependent enhanced Lemaitre model for ductile fracture prediction at low stress triaxiality," Engineering Fracture Mechanics, Vols. 124-125, pp. 80-96, 2014. 
[46] J. R. Rice and D. M. Tracey, "On the ductile enlargement of voids in triaxial stress fields," Journal of the Mechanics and Physics of Solids, vol. 17, pp. 201-217, 1969.

[47] A. A. Benzarga and J. Besson, "Plastic potentials for anisotropic porous solids," European Journal of Mechanics, vol. 20, pp. 397-434, 2001.

[48] Y. Shinohara, Y. Madi and J. Besson, "Anisotropic ductile failure of a high-strength line pipe steel," International Journal of Fracture, vol. 197, pp. 124-145, 2016.

[49] C. Defaisse, M. Mazière, L. Marcin and J. Besson, "Ductile fracture of an ultra-high strength steel under low to moderate stress triaxiality," Engineering Fracture Mechanics, pp. 301-318, 2018.

[50] L. Cortese, T. Coppola, F. Campanelli, F. Campana and M. Sasso, "Prediction of ductile failure in materials for onshore and offshore pipeline applications," International Journal of Damage Mechanics, vol. 23, 2003.

[51] L. Cortese, T. Coppola, F. Campanelli and G. B. Broggiato, "A J2-J3 approach in plastic and damage description of ductile materials," International Journal of Damage Mechanics, vol. 25, 2015.

[52] G. Vadillo, J. Reboul and J. Fernandez-Saez, "A modified Gurson model to account for the influence of the Lode parameter at high triaxialities," European Journal of Mechanics A/Solids, vol. 56, pp. 31-44, 2016.

[53] J. R. Rice, "The localization of plastic deformation," Theoretical and Applied Mechanics, vol. 1, pp. 207-220, 1976.

[54] A. Ranganathan, The Levenberg-Marquardt Algorithm, Computer Science, 8th June 2004. 